\section{Simulation of PV}

Variability As a

Function of PV

Generation and Plant

Size

Aron Habte, Manajit Sengupta, Haoyu Yuan, Grant Buster, and Jin Tan

National Renewable Energy Laboratory, Golden, CO, 80401, USA

$202148^{\text {th }}$ IEEE Photovoltaic Specialists Conference (PVSC), June 20-25, 2021 


\section{Overview}

$>$ Motivation

$>$ Method - Metric used

> National Solar Radiation Database (NSRDB) data

$>$ Result - Baseline analysis

$>$ Result - Using the new method (aggregated short-term variability analysis)

$>$ Summary. 


\section{NSRDB Data}

\section{https://nsrdb.nrel.gov}

Welcome to the

National Solar

Radiation Database 


\section{Motivation}

Some metrics in the literature to quantify short-term photovoltaic (PV) variability provide limited direct insight to guide power system operation and/or planning.

* This study focuses on a new metric that reflects the aggregated PV variability, which can be used as an indicator of PV variability's impact on system operation.

* The new metric could also assist engineers in power system planning to understand the system-level distribution of variability with an option to select various time horizon levels of variability to perform system studies.

* Forecasting such a metric in various timescales will be essential to system operators for dispatch optimization. 


\section{Normalized Variability Metric}

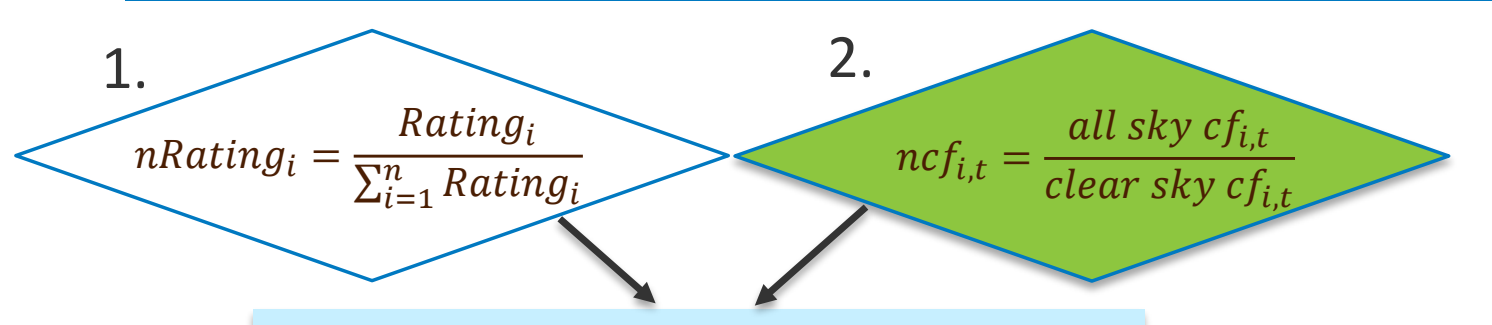

1. Each plant rating was used in the analysis, and normalized rating was calculated.

2. All-sky and clear-sky capacity factors were used to generate the normalized capacity factor for each time series of each plant.

3. $\Delta$ Normalized $\mathrm{CF}_{i, t}=\left(\Delta n c f_{i, \Delta t}\right) *$ nRating $_{i}$

3. The difference in each consecutive time step (timescale) was calculated and then multiplied by the normalized rating (Step 1).

4. $\Delta$ Normalized $\mathrm{CF}_{t}=\sum \Delta$ Normalized $\mathrm{CF}_{i, t}$

4. All the plants' output from Step 3 was summed to get one array of a time-series data set.

Variability Statistics:

5. Variability statistics were calculated for the duration of interest (daily or hourly).

5. 1. Standard deviation for a duration (e.g., daily or hourly)

2. Clear and cloudy index (average of $n c f_{t}$ ).

- The normalized metric varies from [0,1], with 0 representing no variability, such as a clearsky condition. It captures the cloud impact.

- Timescale is determined by $\Delta t$.

- Duration is the range within which statistics are calculated. 


\section{Baseline Analysis}

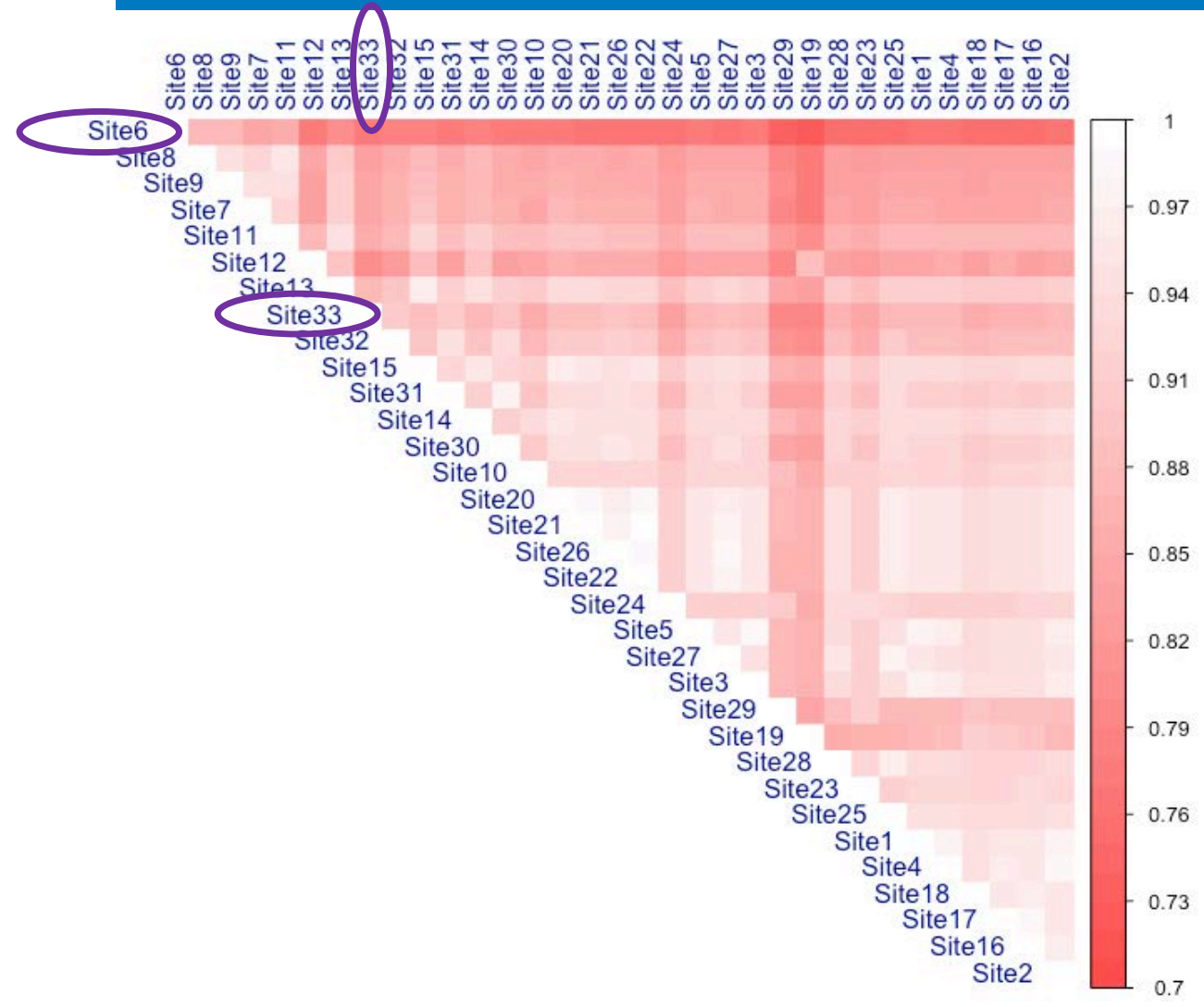

- The results of the correlation matrix are consistent with those in the previous slide.

- It is not always true that spatial correlation among sites varies inversely with distance.

Site 6 is relatively far ( 55 km) from Site 33, but the correlation between them is higher than for some of the closest sites to Site 6, which are within $15 \mathrm{~km}$. 


\section{Aggregated Short-Term Variability Analysis}

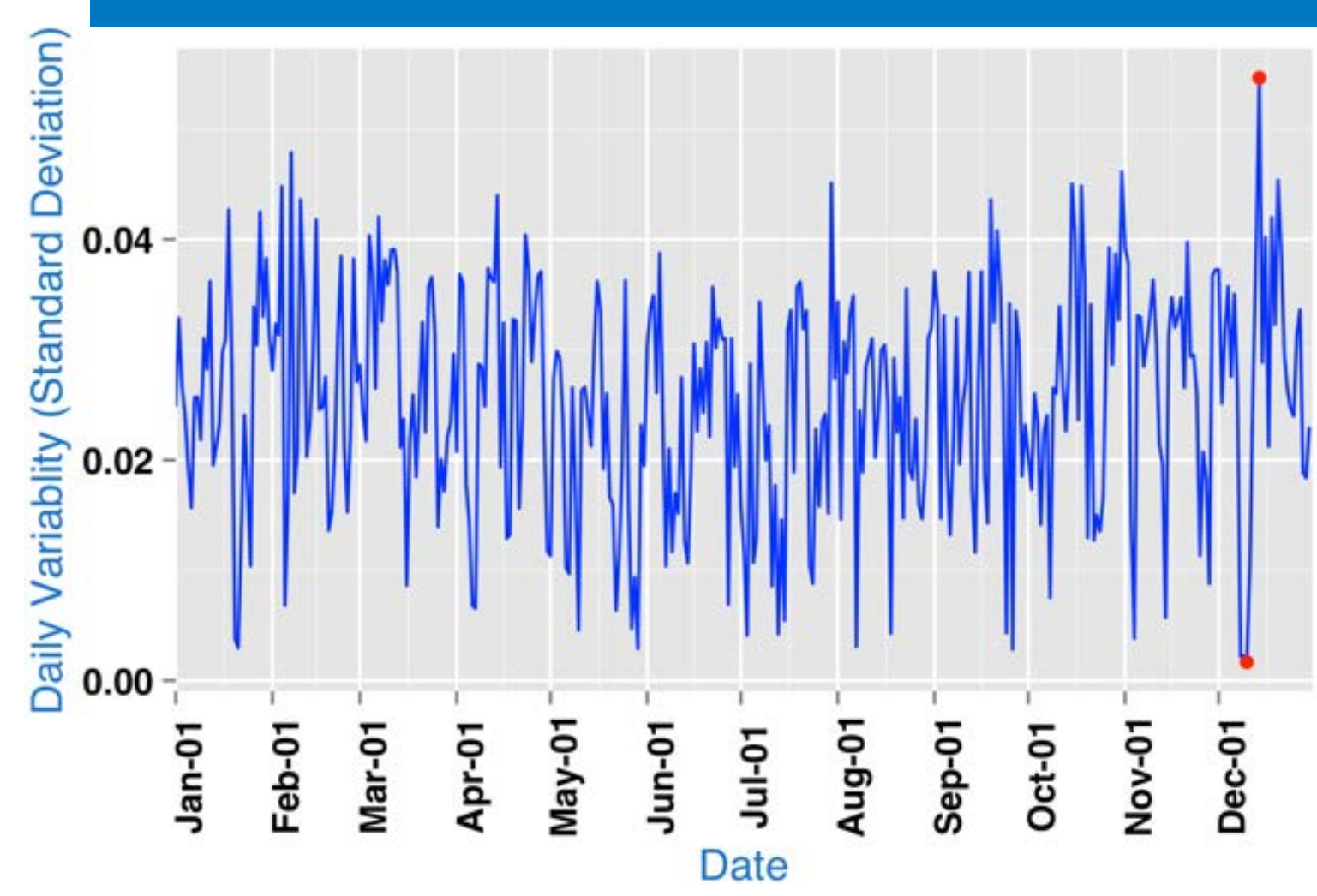

Nominal daily variability aggregated from the 1-minute data. The red points show the maximum and minimum variability, which coincidentally both occur during December 2019. 


\section{Aggregated Short-Term Variability Analysis}

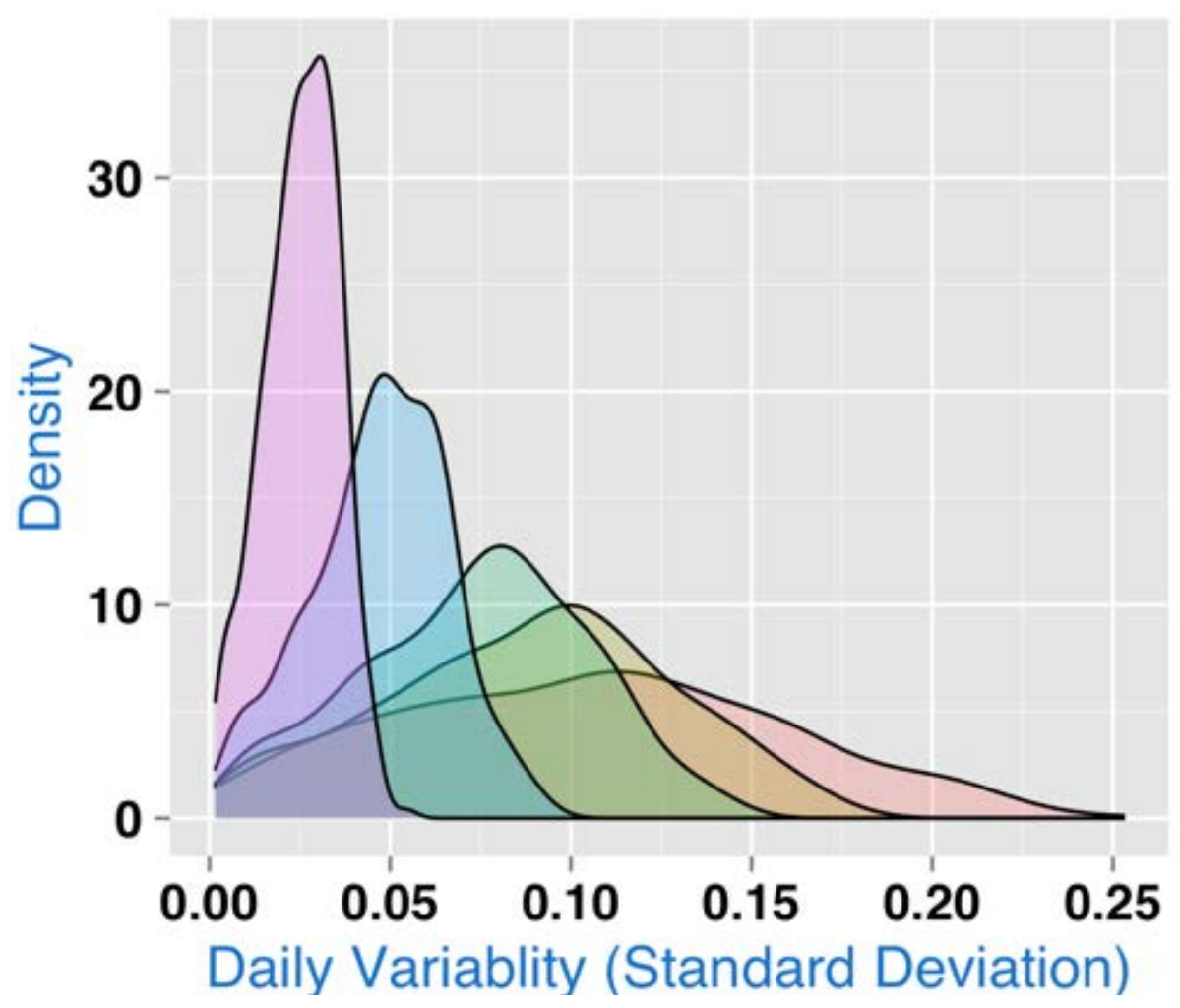

- Analysis can be undertaken using various other time

Time scale

$\square$ 60-min 30-min 15-min 5-min 1-min resolutions, namely, with 5-, 15-, 30-, and 60-minute timescales.

- On a daily basis, hourly intervals induce higher aggregated ramp rates than the other timescales. 


\section{Summary}

- Short term fluctuation in solar generation from PV cause challenges for system operations.

- These events need to be understood clearly to mitigate their effects on system operations, both for individual systems and fleets of systems at the regional scale.

- Using solar resource data from 33 sites within a relatively small-but highly inhomogeneous - region, this study provided a method to quantify these effects using a new statistical metric.

- One important finding was that the correlation between the output of different systems was not solely conditioned by their distance, contrary to what some current literature suggests. 


\section{Thank you!}

\section{Email: aron.habte@nrel.gov}

\section{www.nrel.gov}

NREL/PR-5D00-80237

This work was authored by the National Renewable Energy Laboratory, operated by Alliance for Sustainable Energy, LLC, for the U.S. Department of Energy (DOE) under Contract No. DE-AC36-08GO28308. Funding provided by the U.S. Department of Energy Office of Energy Efficiency and Renewable Energy Solar Energy Technologies Office Award Number 34224. The views expressed in the article do not necessarily represent the views of the DOE or the U.S. Government. The U.S. Government retains and the publisher, by accepting the article for publication, acknowledges that the U.S. Government retains a nonexclusive, paid-up, irrevocable, worldwide license to publish or reproduce the published form of this work, or allow others to do so, for U.S. Government purposes. 